\title{
Optical Characterization of Porous Sputtered Silver Thin Films
}

\author{
Olivier Carton, ${ }^{1,2}$ Jaouad Ghaymouni, ${ }^{1}$ Michaël Lejeune, ${ }^{1}$ and Andreas Zeinert ${ }^{1}$ \\ ${ }^{1}$ Laboratoire de Physique de la Matière Condensée, Université de Picardie Jules Verne, 33 rue Saint Leu, \\ 80039 Amiens Cedex 1, France \\ ${ }^{2}$ Laboratoire de Mécanique Roberval, Université de Technologie de Compiègne, Centre de Recherches de Royallieu, \\ 60203 Compiègne Cedex, France
}

Correspondence should be addressed to Olivier Carton; o_carton@hotmail.com

Received 15 May 2013; Revised 9 September 2013; Accepted 10 September 2013

Academic Editor: Damien Boyer

Copyright (c) 2013 Olivier Carton et al. This is an open access article distributed under the Creative Commons Attribution License, which permits unrestricted use, distribution, and reproduction in any medium, provided the original work is properly cited.

The optical properties of various porous silver films, grown with a commercial DC sputter coater, were investigated and compared for different plasma parameters. Effective Drude models were successfully used for those films whose spectra did not show particular resonance peaks. For the other films, neither an effective Drude model nor effective medium models (Maxwell Garnett, Bruggeman, and Looyenga) can describe the optical properties. It turns out that a more general approach like the Bergman representation describes the optical data of these films accurately adopting porosity values consistent with physical measurements.

\section{Introduction}

Silver (Ag) thin films are widely used in transparent and heat reflecting layers stacks of the solar-control or low-e type, applied in glazing units for buildings and automobiles as well as for solar energy engineering purposes for passive heat gain $[1,2]$. The Ag thin films used in these kinds of optical systems are typically deposited by magnetron sputtering. It is well known that $\mathrm{Ag}$ films with good optical properties (high reflection and low absorption) can be obtained with this technique [1-6]. Like for almost metals silver film growth starts with separated islands, which spread and percolate with increasing thickness. At this early stage the absorption of visible light is higher than the one of bulk silver. When a critical actual thickness $d_{c}$ is reached, the film will show the optical properties of the bulk [7-10]. Several authors have shown that the deposition parameters highly influence this film growth and as a consequence the dielectric function of the material $[7,11,12]$. However all these investigations focus on films which reach optical Ag bulk properties after a critical thickness $d_{c}$ around only $10-20 \mathrm{~nm}$. In this paper we have deposited two series of silver films. The first series A concerns films with higher $d_{c}$ (between 50 and $100 \mathrm{~nm}$ ). Series B of films was grown under plasma conditions leading to materials that do not reach optical Ag bulk behavior, until very high thickness values up to $1.38 \mu \mathrm{m}$. All these films can be deposited with a simple commercial coater. The plasma conditions used with this technique allow a film growth with a large range of optical properties: coatings with a high reflectance and a low absorption in the visible range as well as porous films exhibiting a low reflectance and a high absorption. Porous metal films can be considered as composites with an admix of the metal phase and voids. To describe the optical properties of these materials usually an effective refractive index is proposed. Different models are mentioned in the literature, depending on the more or less complex microstructure of the films. An optical modeling procedure that defines response at all stages of growth has been developed. For silver an admix of three distinct types of plasmonic responses is adequate, two are localized, and one is delocalized $[13,14]$. Another procedure is based on a varying spectral density representation [15]: the Bergman representation [16]. This spectral density is linked to the topology of the studied material. For some particular topologies, this representation can be simplified and gives the equations of the common effective medium theories (Maxwell-Garnet, Bruggeman, and Looyenga) [17-19]. In this paper we will show that in some cases only the more general but much less frequently used Bergman representation can account for the experimental data. This study is not focused 
on the quality and the use of the obtained silver coatings, its aim is to show the suitability of different optical models which must be used to extract the properties of silver films with different structures.

\section{Materials and Methods}

Silver thin films have been deposited on glass substrate by magnetron DC sputtering from a pure silver target (diameter $5.7 \mathrm{~cm}$ ) using a commercial sputter coater (Cressington 108 auto; this kind of coater is commonly used for metal coatings for electrical contacts and also in some cases for SEM observations). The based pressure in the vacuum chamber (around $3 \mathrm{~Pa}$ ) was obtained with a single rotary pump. Argon was used as sputtering gas, and the work pressure was between 3.5 and $10 \mathrm{~Pa}$. The direct current (DC) applied was in the range from 10 to $40 \mathrm{~mA}$. Optical measurements have been performed after deposition with a Cary $5 \mathrm{E}$ spectrophotometer using an integrating sphere in order to take into account any scattering of light induced by the surface roughness of the films. The commercial optical thin film software CODE [21] has been used to fit the reflectance and transmittance curves obtained for each sample. Films physical thickness was measured with a Dektak 3ST profilometer. The porosity of the films has been evaluated by measuring the weight of the substrate before and after the deposition of the coating (the sensitivity of the balance was $10 \mu \mathrm{g}$ ). The density of the samples was then calculated and compared to the theoretical density of silver $\left(10.5 \mathrm{~g} \cdot \mathrm{cm}^{-3}\right)$. The thickness of the porous films studied was at least $190 \mathrm{~nm}$ in order to minimize the experimental error which is around $10 \%$.

\section{Results and Discussion}

Previous studies revealed that the most important growth parameters of sputtered silver thin films are the pressure and the power. These two parameters determine the critical thickness $d_{c}$ of the films which influences the optical properties. We have investigated the effects of these parameters on our Ag thin films. The curves in Figure 1 show the evolution of the refractive index $n$ and the extinction coefficient $k$ for different work pressures and currents. These curves have been obtained by fitting the experimental reflectance and transmittance spectra, using an effective Drude model considering a homogeneous film on a glass substrate. Except for the film deposited at $40 \mathrm{~mA}$ and $3.5 \mathrm{~Pa}$ with a thickness of $106 \mathrm{~nm}, n$ and $k$ are different compared to the known bulk values [22].

As already shown by several authors [2, 4, 6, 7], a higher power and a lower pressure lead to a decrease of $n$ and an increase of $k$ approaching the bulk data. Several groups have proposed an explanation for the observed spread for $n$ and $k$ compared to the bulk: when the films are in the first step of growth, the presence of surface plasmons will determine the optical constants $[1,23,24]$. Three types of plasmonic response will occur during the film growth: the first is due to groups of isolated silver nanoislands initially present on the substrate, then another will be due to single silver islands
TABLE 1: Effective plasma frequency depending on the deposition parameters.

\begin{tabular}{lccc}
\hline Current $(\mathrm{mA})$ & Pressure (Pa) & Thickness (nm) & $\begin{array}{c}\text { Plasma } \\
\text { Frequency }(\mathrm{eV})\end{array}$ \\
\hline 20 & 3.5 & 22 & 5.55 \\
40 & 8 & 22 & 5.74 \\
40 & 3.5 & 26 & 7.38 \\
40 & 3.5 & 50 & 8.04 \\
40 & 3.5 & 106 & 8.91 \\
\hline
\end{tabular}

surrounded but separated from the conducting network, and the last due to the small scale network which is represented by a Drude-like response [14]. The films will exhibit optical properties of the bulk only when the critical thickness $d_{c}$ is reached.

The effective Drude model used gives an effective plasma frequency for each sample (Table 1). This effective plasma frequency is linked to the porosity of the material $[13,14,25]$ Depending on the plasma parameters, when the effective constants of the films approach the ones of the Ag bulk (i.e., when the pressure decreases and the thickness and the current increase), the effective plasma frequency increases to reach the silver bulk plasma frequency (9.04 eV [26]). Our results indicate that some voids are present in the films until the critical thickness is reached.

In order to understand the influence of power and pressure on films properties, the growth mechanism of silver has been explained as follows: the power and the pressure influence the surface diffusion of Ag atoms through the input energy into the growing film and through the growth rate [11]. When the power decreases and the pressure increases, the injected energy is higher [27], while the growth rate is lower, implying a higher surface diffusion. Surface diffusion enhances the formation of islands in the first step of growth and as a consequence delays the formation of a homogeneous film; consequently the critical thickness $d_{c}$ will increase. In the literature, for based pressures lower than $2 \cdot 10^{-3} \mathrm{~Pa}$ and work argon pressures between 0.1 and $2.5 \mathrm{~Pa}$, the critical thickness is around $10-20 \mathrm{~nm}$ [4-7]. In our case our base pressure is as high as $3 \mathrm{~Pa}$, whereas the working pressure is in the range from 3.5 to $8 \mathrm{~Pa}$; therefore one can expect higher values of $d_{c}$. As seen in Figure 1, even with the highest available current $(40 \mathrm{~mA})$ and the lowest pressure (3.5 Pa), $d_{c}$ lies between 50 and $100 \mathrm{~nm}$ (we assumed that $d_{c}$ is reached when the optical constants of the films are similar to the one of the Ag bulk). This means that even in these "optimized" conditions available for this kind of coater, the island formation is predominant and strongly delays the formation of a homogeneous film. One can expect that by selecting an opposite condition with a high pressure, $d_{c}$ will drastically increase or even never be reached. This last case was explored in the present work with the films of series B. We have deposited films of different thickness (190, 750, and $1380 \mathrm{~nm}$ ) with the maximum available pressure around $10 \mathrm{~Pa}$ in order to promote porosity. The rather high current of $40 \mathrm{~mA}$ was chosen in order to prevent silver target heating 

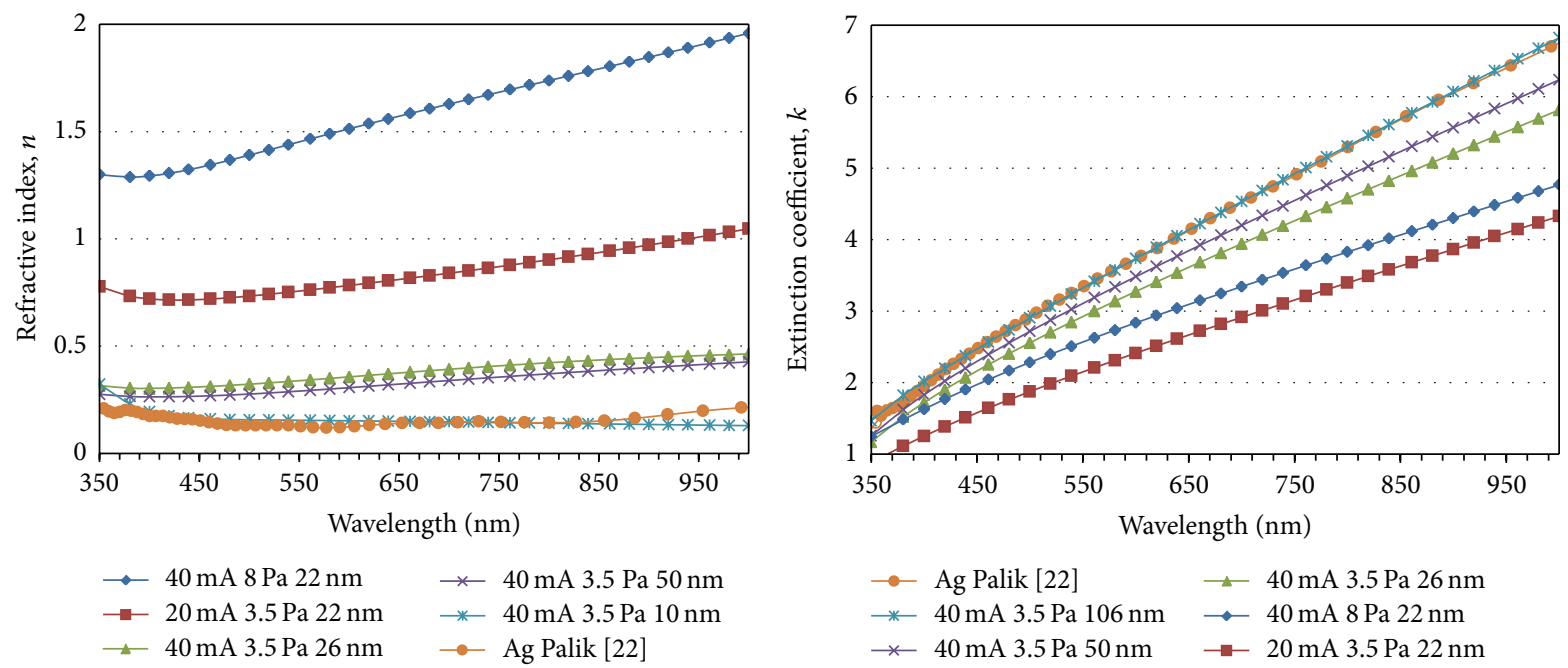

FIGURE 1: Refractive index and extinction coefficient obtained by fitting the reflectance and transmittance spectra, using an effective Drude model considering a homogeneous film.

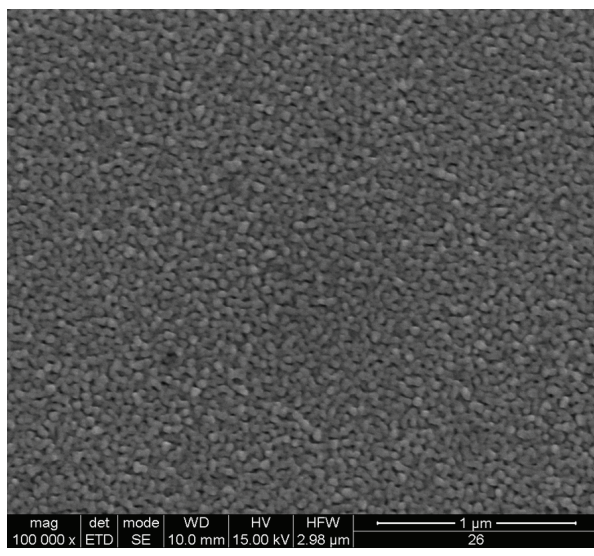

(a)

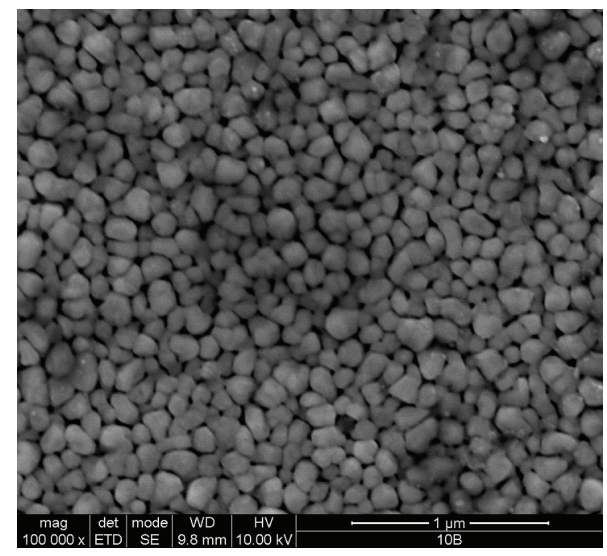

(b)

Figure 2: SEM images of silver films deposited at $40 \mathrm{~mA}$ and 10 Pa for different physical thicknesses: (a) 190 and (b) $1380 \mathrm{~nm}$.

(with our coater there is no water cooling system of the cathode) and keep the deposition time acceptable (about 70 minutes for the $1383 \mathrm{~nm}$ thick film). In such conditions, the surface of the coating is not smooth but is composed by silver grains of different sizes, as can been seen on the picture obtained by scanning electron microscopy (Figure 2 ).

The size of the silver grains increases with the film thickness. Due to this morphology the measured porosity of the coatings is high: 27,38 , and $36 \%$, respectively, for the 190, 750, and $1380 \mathrm{~nm}$ thick films. In contrast to series A described before (Figure 1), an effective Drude component cannot fit the obtained reflectance spectra of series B. Thus other models have to be tested to describe the optical properties of these films whose void-metal phase topology seems to favor multiple resonances which are still present in the spectra. Effective medium approximations seem to be tempting approaches for describing these spectra and get an effective optical index of these materials. Several different models have been tested and compared: the "simple mixing models" of Maxwell Garnett [17], Bruggeman [18], and Looyenga [19]. One can expect the possibility that these "simple" models are not adequate regarding the diversity of structures involved in our coatings, because they are all based on special assumptions about sample topology (particles are generally considered as spheres or ellipsoids). Thus we compared the results obtained with these models to the ones obtained with a more general procedure based on a varying spectral density: the Bergman representation [16].

Within the effective medium approximation we consider a volume fraction $f$ of particles with a dielectric function $\varepsilon$ embedded in a matrix $\varepsilon_{M}$. In our case $\varepsilon$ represents the dielectric function of voids in a silver matrix with $\varepsilon_{M}$. The dielectric function $\varepsilon_{\text {eff }}$ of the considered effective medium is defined by the following formulae.

Maxwell Garnett:

$$
\frac{\varepsilon_{\mathrm{eff}}-\varepsilon_{M}}{\varepsilon_{\mathrm{eff}}+2 \varepsilon_{M}}=f \frac{\varepsilon-\varepsilon_{M}}{\varepsilon+2 \varepsilon_{M}}
$$




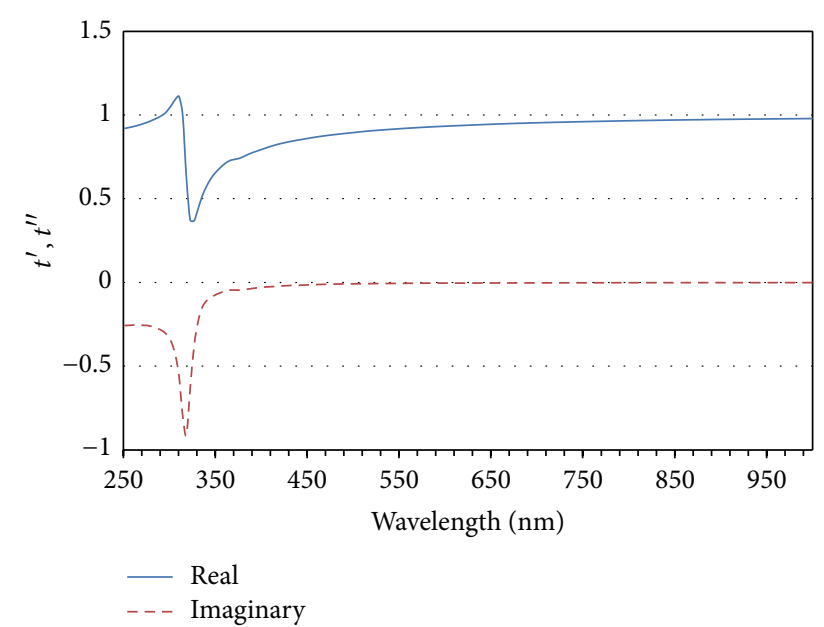

FIGURE 3: Function $t$ of the Bergman representation plotted considering voids embedded in a silver matrix.

Bruggeman:

$$
f \frac{\varepsilon-\varepsilon_{\mathrm{eff}}}{\varepsilon+2 \varepsilon_{\mathrm{eff}}}+(1-f) \frac{\varepsilon_{M}-\varepsilon_{\mathrm{eff}}}{\varepsilon_{M}+2 \varepsilon_{\mathrm{eff}}}=0 .
$$

Looyenga:

$$
\varepsilon_{\mathrm{eff}}^{1 / 3}=f \varepsilon_{\mathrm{eff}}^{1 / 3}+(1-f) \varepsilon_{M}^{1 / 3}
$$

Bergman:

$$
\varepsilon_{\mathrm{eff}}=\varepsilon_{M}\left(1-f \int_{0}^{1} \frac{g(n, f)}{t-n} d n\right)
$$

with

$$
t=\frac{\varepsilon_{M}}{\varepsilon_{M}-\varepsilon}
$$

$g(n, f)$ represents the spectral density which is a function depending only on the topology of the phase mixture and independent of the respective dielectric functions of the two considered phases (silver and vacuum). This function is normalized:

$$
\int_{0}^{1} g(n, f) d n=1 .
$$

To investigate the utility of using the Bergman representation, we plotted the real and the imaginary parts $t^{\prime}$ and $t^{\prime}$ of the function $t$ in the studied spectral range [28], using the optical constant $\varepsilon_{M}$ from [22] for silver, considering voids embedded in a silver matrix (Figure 3 ).

Because the real part of $t$ is close to the interval $[0,1]$ the dielectric properties of our coatings are sensitive to the topology [29]. Thus the Bergman representation seems more appropriate to model these properties, and a spectral density has to be adjusted to the experimental data for each film, in addition to film thickness and volume fraction $f$.
As an example, we show a comparison of the fits obtained with the different effective medium theories for the $1380 \mathrm{~nm}$ thick film with a measured porosity of $36 \%$ (Figure 4 ).

In the first case (Figure 4(a)) the thickness and the porosity of the coating are imposed and only the reflectance curve is fitted. In the second case (Figure 4(b)), the thickness and the porosity are now free parameters in the models (and the spectral density for the Bergman representation). The best fits obtained with the Maxwell-Garnett, Bruggeman, and Looyenga models exhibit a large deviation and are not acceptable, and in the second case the thickness and the porosity given are, respectively, $1250 \mathrm{~nm}$ and 75\%, $1550 \mathrm{~nm}$ and $45 \%$, and 1400 and 35\%. Only the Bergman representation is suitable for this coating, because the fit obtained is excellent and the thickness and the porosity (1400 and $36 \%$ ) are in good agreement with the experimental measures. We obtain the same behavior for our other two coatings (190 and $750 \mathrm{~nm}$ ): "simple mixing models" fail, whereas the Bergman representation is suitable. As already mentioned, the "simple" models failed mainly because of the complexity of the topologies of the sample which leads to multiple plasmon resonances. These models are based on specific assumptions about sample topology.

The effective optical constants obtained for these three investigated silver films with the Bergman representation are plotted in Figure 5. We can note the characteristic signature of a plasmon around $380 \mathrm{~nm}$ on the refractive index curves of the thicker and most porous films, whereas it is less pronounced for the thinner film which is denser and thus more homogeneous. For wavelengths below the plasmon resonance the constants become closer to the one of the bulk [22]. It is likely that other resonances are present but not distinguishable on the imaginary part of the refractive index spectra for higher wavelengths. As already described in the literature [13], due to the complexity of the topology of the films, involving many different shapes of particles, the contribution of the different resonances can lead to a "simple" curve for $k$ washing out the individual absorption peaks, quite parallel to the one of the Ag bulk.

As explained above, the spectral density $g(n, f)$ depends on the topology of the mixture. As in our case the porosity and the Ag grain size change with thickness, and a different topology of the films is expected and consequently $g$ will also vary. This is indeed observed in Figure 6.

The link between these curves and the topology of the mixture is not straightforward. Following the conclusions given in [20], where porous $\mathrm{SiO}_{2}$ was investigated, an interpretation can be tried. The broad peak around $n=0.15$ corresponds to isolated silver particles of the matrix, and the peak closer to 0 represents connected silver particles. These suggestions are in good agreement with our results because as the porosity of the layer increases, the particles of silver are more isolated and the percolation becomes lower. The analysis suggests a network connection in the films. The SEM images (Figure 2) of these coatings cannot show precisely the percolation, but while column growth is favouring voids, this does not exclude that the columns touch, and all the more the thickness of the films is quite high. 


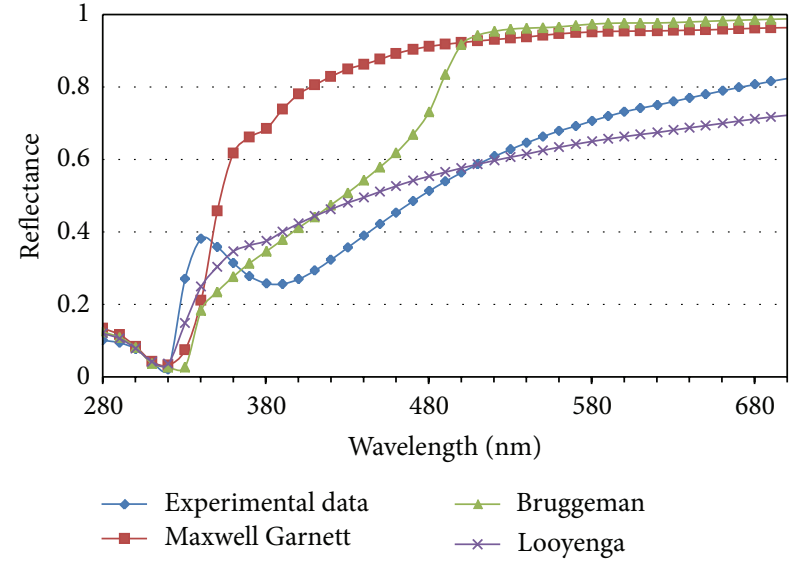

(a)

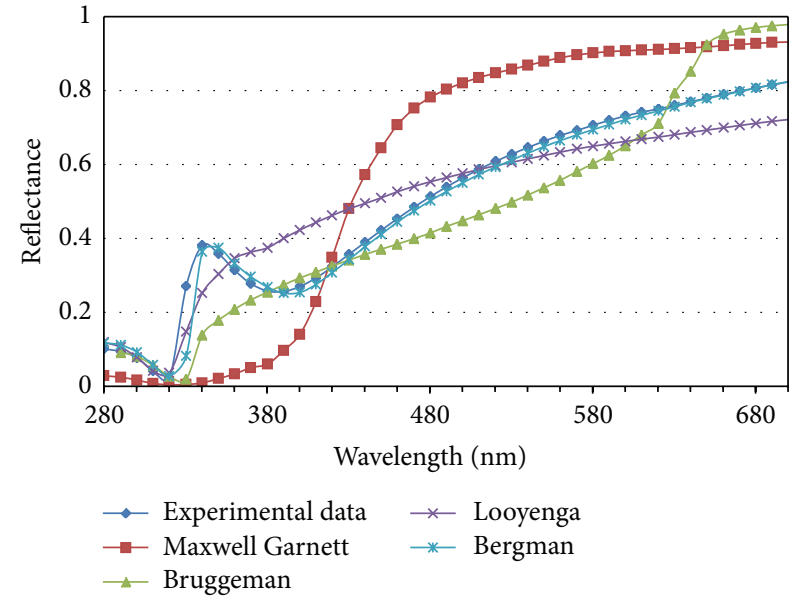

(b)

FIGURE 4: Fit of the reflectance curve of the $1380 \mathrm{~nm}$ thick and 36\% porous silver film with the different effective medium theories: (a) thickness of $1380 \mathrm{~nm}$ and porosity of $36 \%$ are fixed in the models; (b) experimental parameters are unconstrained.
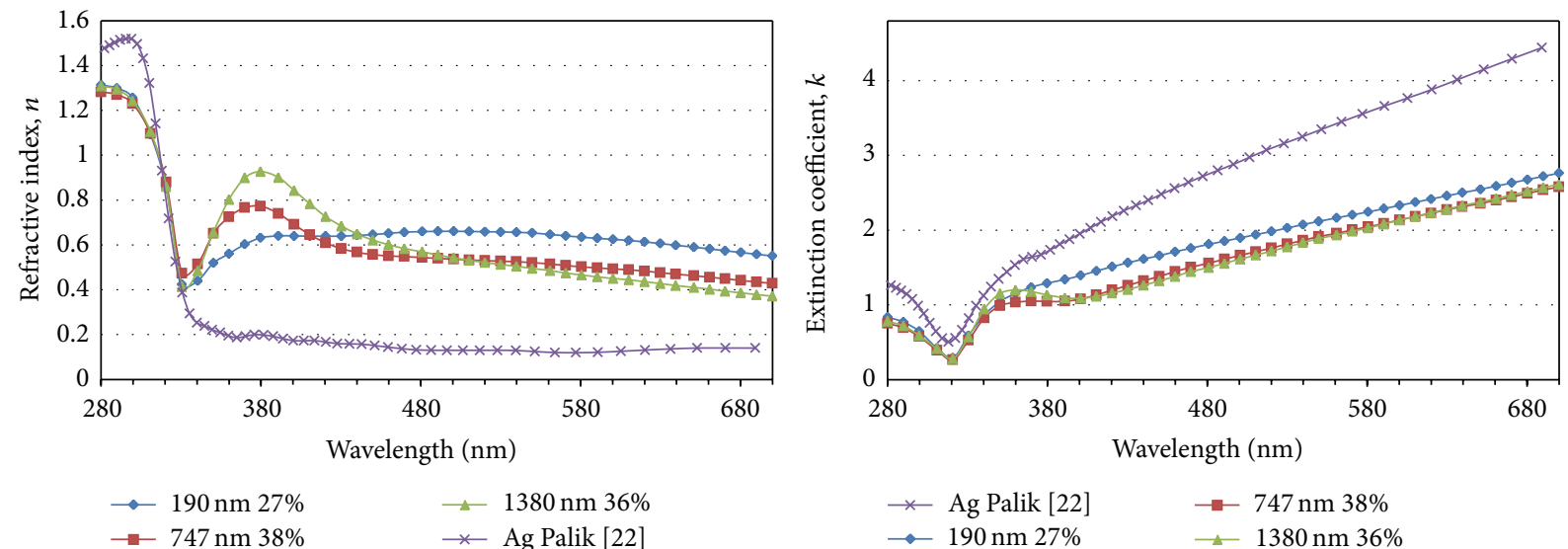

FIGURE 5: Effective refractive index and extinction coefficient obtained for series B of Ag films with the Bergman representation.

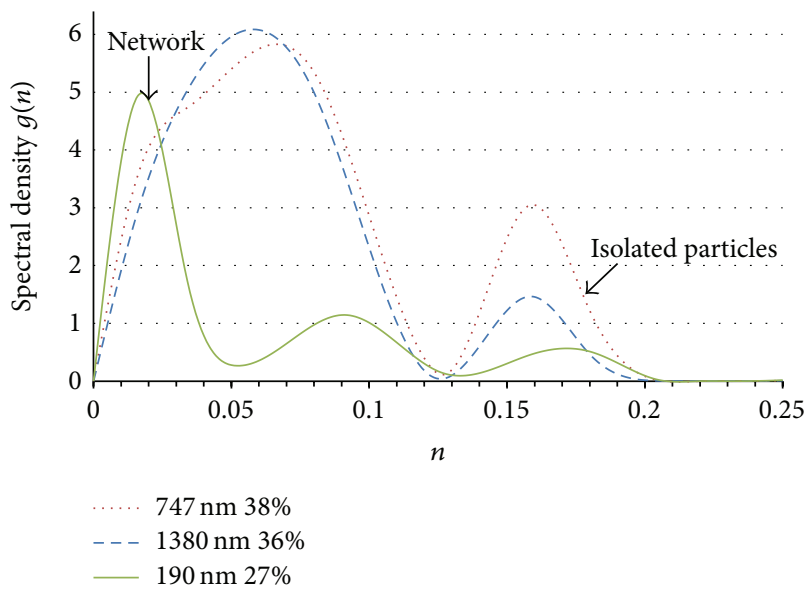

FIGURE 6: Spectral densities in the interval [0, 0.25] obtained for our silver thin films and interpretation according to [20].

\section{Conclusions}

Silver thin films have been deposited with a commercial sputter coater. We have shown that, depending on the plasma parameters and consequently on the growth of the film, it is possible to obtain films with different optical properties: when the plasma conditions limit surface diffusion during growth, the films exhibit Drude-like behaviour and the spectra can be fitted by an effective Drude model and the lower plasma frequency indicates film porosity. For a sufficient high physical film thickness one approaches the optical properties of bulk Ag and the effective model converges to the Ag bulk Drude model. In this last case the coating technique provides good optical properties with bulk Ag characteristics. However if the power is decreased while pressure is increased, surface diffusion is strongly favored and prevents the films from reaching Ag bulk even for very high thicknesses. The optical spectra of these films cannot be described by an 
effective Drude model in the visible range, neither can they be fitted by standard effective medium approaches using "simple mixing formulas" like Maxwell Garnett, Bruggeman, and Looyenga. The form of the spectra and the film porosity rate (independently extracted by physical measurements) can only be correctly described by the effective medium Bergman representation which can take into account the remaining plasmon resonances due to the particular metalvoid phase topology of these films [29]. While this porous topology is difficult to grasp with conventional techniques an interpretation of the link between the curves obtained for the spectral densities and the topology of the coatings has been proposed, in agreement with the work of another group who used the same model for a different material.

\section{Conflict of Interests}

The authors certify that they do not have a direct financial relation with the commercial identity mentioned in this paper. There is no conflict of interests for any of the authors.

\section{Acknowledgment}

This work is supported by the MICHROB project funded by the Conseil Régional de Picardie, France.

\section{References}

[1] P. Zhao, W. Su, R. Wang, X. Xu, and F. Zhang, "Properties of thin silver films with different thickness," Physica E, vol. 41, no. 3, pp. 387-390, 2009.

[2] C. Charton and M. Fahland, "Optical and electrical properties of sputtered Ag films on PET webs," Surface and Coatings Technology, vol. 142-144, pp. 175-180, 2001.

[3] T. Suzuki, Y. Abe, M. Kawamura, K. Sasaki, T. Shouzu, and K. Kawamata, "Optical and electrical properties of pure Ag and Ag-based alloy thin films prepared by RF magnetron sputtering," Vacuum, vol. 66, no. 3-4, pp. 501-504, 2002.

[4] X. Sun, R. Hong, H. Hou, Z. Fan, and J. Shao, "Thickness dependence of structure and optical properties of silver films deposited by magnetron sputtering," Thin Solid Films, vol. 515, no. 17, pp. 6962-6966, 2007.

[5] G. Xu, M. Tazawa, P. Jin, and S. Nakao, "Surface plasmon resonance of sputtered Ag films: Substrate and mass thickness dependence," Applied Physics A, vol. 80, no. 7, pp. 1535-1540, 2005.

[6] Y. S. Jung, "Study on texture evolution and properties of silver thin films prepared by sputtering deposition," Applied Surface Science, vol. 221, no. 1-4, pp. 281-287, 2004.

[7] C. Charton and M. Fahland, "Optical properties of thin Ag films deposited by magnetron sputtering," Surface and Coatings Technology, vol. 174-175, pp. 181-186, 2003.

[8] F. Abelès, Y. Borensztein, and T. López-Rios, "Optical properties of discontinuous thin films and rough surfaces of silver," Festkörperprobleme, vol. 24, pp. 93-117, 1984.

[9] T. Robin and B. Souillard, "Long wavelength properties of granular metal-insulator films: a microscopic approach," Physica A, vol. 157, no. 1, pp. 285-292, 1989.

[10] R. R. Singer, A. Leitner, and F. R. Aussenegg, "Structure analysis and models for optical constants of discontinuous metallic silver films," Journal of the Optical Society of America B, vol. 12, no. 2, pp. 220-228, 1995.

[11] C. Charton and M. Fahland, "Growth of Ag films on PET deposited by magnetron sputtering," Vacuum, vol. 68, no. 1, pp. 65-73, 2002.

[12] M. Del Re, R. Gouttebaron, J. P. Dauchot et al., "Growth and morphology of magnetron sputter deposited silver films," Surface and Coatings Technology, vol. 151-152, pp. 86-90, 2002.

[13] G. B. Smith and A. A. Earp, "Metal-in-metal localized surface plasmon resonance," Nanotechnology, vol. 21, no. 1, Article ID 015203, 2010.

[14] A. A. Earp and G. B. Smith, "Evolution of plasmonic response in growing silver thin films with pre-percolation non-local conduction and emittance drop," Journal of Physics D, vol. 44, no. 25, Article ID 255102, 2011.

[15] E. Tuncer and G. A. Niklasson, "Optical properties of non-dilute metal-insulator composites," Optics Communications, vol. 281, no. 17, pp. 4374-4379, 2008.

[16] D. J. Bergman, "The dielectric constant of a composite materialA problem in classical physics," Physics Reports, vol. 43, no. 9, pp. 377-407, 1978.

[17] J. C. Maxwell Garnett, "Colours in metal glasses and in metallic films," Philosophical Transactions of the Royal Society of London A, vol. 203, pp. 385-420, 1904.

[18] D. A. G. Bruggeman, "Berechnung verschiedener physikalischer konstanten von heterogenen substanzen," Annals of Physics, vol. 24, pp. 636-679, 1935.

[19] H. Looyenga, "Dielectric constants of heterogeneous mixtures," Physica, vol. 31, no. 3, pp. 401-406, 1965.

[20] W. Theiss, S. Henkel, and M. Arntzen, "Connecting microscopic and macroscopic properties of porous media: choosing appropriate effective medium concepts," Thin Solid Films, vol. 255, no. 1-2, pp. 177-180, 1995.

[21] W. Theiss Hard- and Software, http://www.wtheiss.com/.

[22] E. D. Palik, Handbook of Optical Constants of Solids, Academic Press, San Diego, Calif, USA, 1998.

[23] A. I. Maaroof and G. B. Smith, "Effective optical constants of nanostructured thin silver films and impact of an insulator coating," Thin Solid Films, vol. 485, no. 1-2, pp. 198-206, 2005.

[24] G. B. Smith and A. I. Maaroof, "Optical response in nanostructured thin metal films with dielectric over-layers," Optics Communications, vol. 242, no. 4-6, pp. 383-392, 2004.

[25] A. A. Earp and G. B. Smith, "Metal nanoparticle plasmonics inside reflecting metal films," Applied Physics Letters, vol. 96, no. 24, Article ID 243108, 2010.

[26] E. J. Zeman and G. C. Schatz, "An accurate electromagnetic theory study of surface enhancement factors for $\mathrm{Ag}, \mathrm{Au}, \mathrm{Cu}, \mathrm{Li}$, $\mathrm{Na}, \mathrm{Al}, \mathrm{Ga}$, In, Zn, and Cd," Journal of Physical Chemistry, vol. 91, no. 3, pp. 634-643, 1987.

[27] T. P. Drüsedau, T. Bock, T.-M. John, F. Klabunde, and W. Eckstein, "Energy transfer into the growing film during sputter deposition: an investigation by calorimetric measurements and Monte Carlo simulations," Journal of Vacuum Science and Technology A, vol. 17, no. 5, pp. 2896-2905, 1999.

[28] W. Theiss, "The use of effective medium theories in optical spectroscopy," Advances in Solid State Physics, vol. 33, pp. 149$176,1994$.

[29] J. Sturm, P. Grosse, and W. Theiss, "Effective dielectric functions of alkali halide composites and their spectral representation," Zeitschrift für Physik B Condensed Matter, vol. 83, no. 3, pp. 361$365,1991$. 

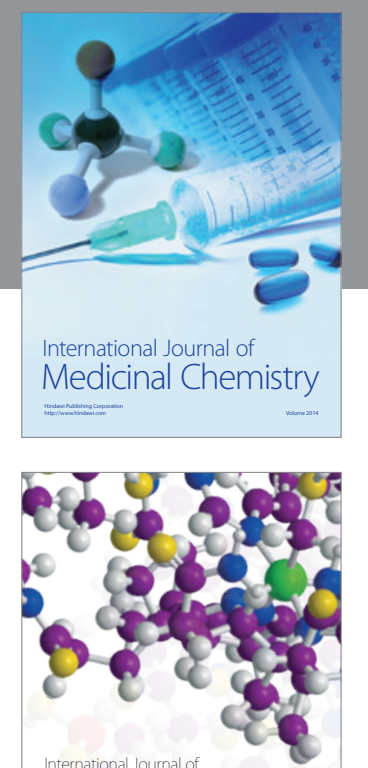

\section{Carbohydrate} Chemistry

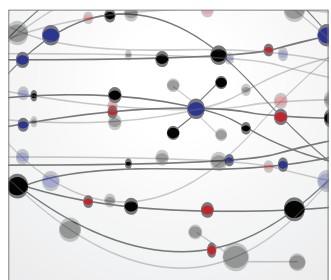

The Scientific World Journal
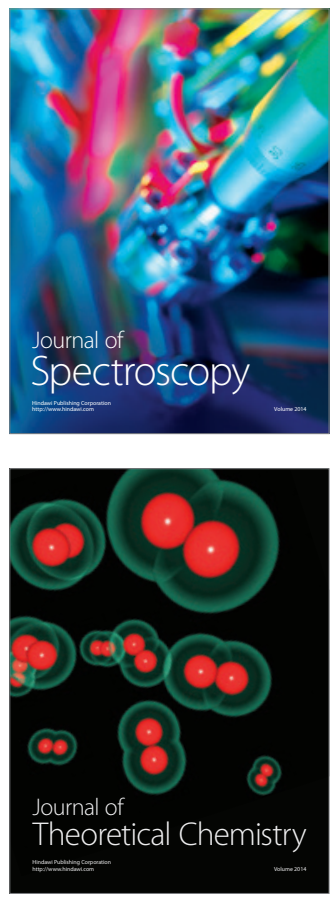
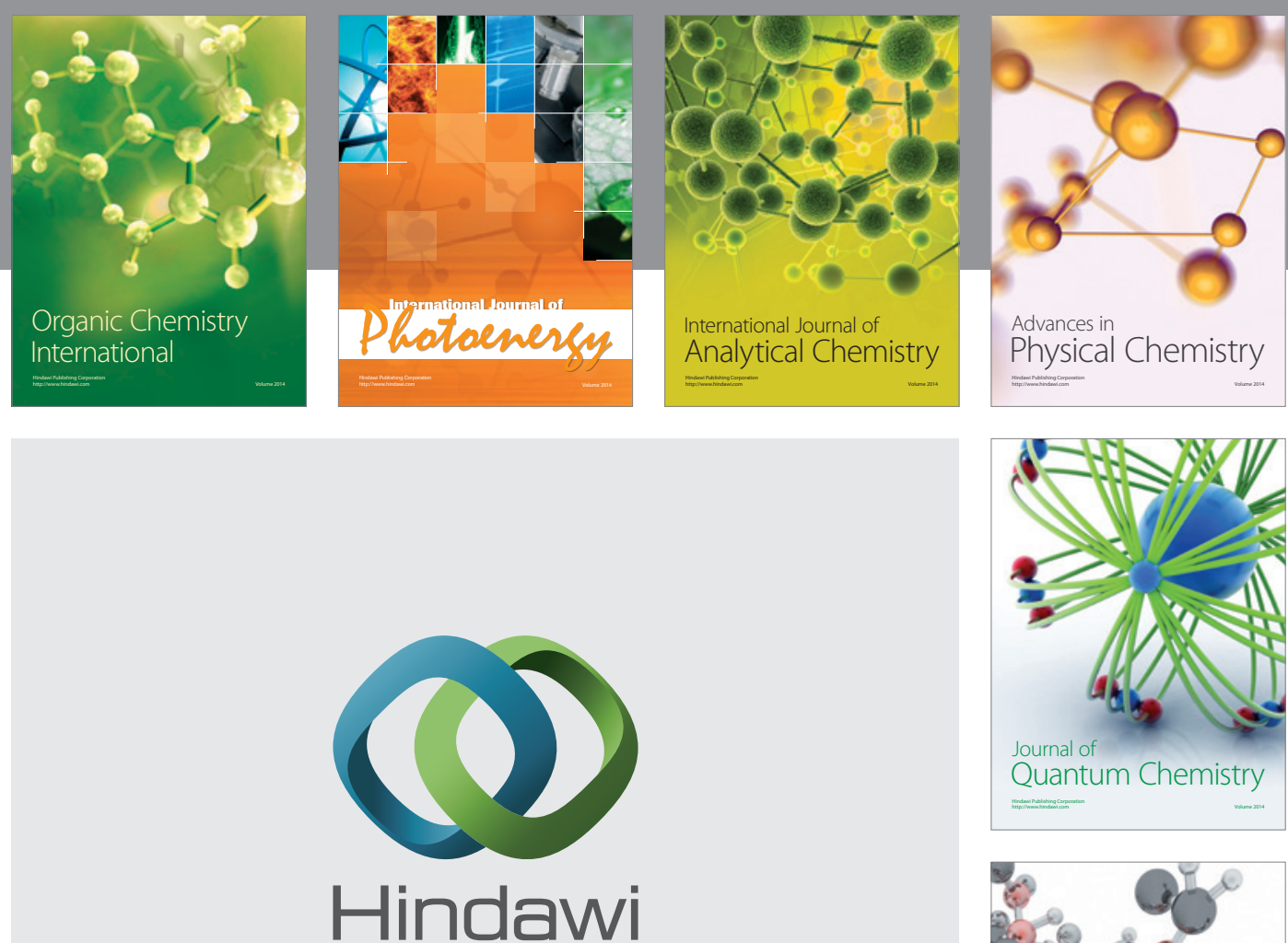

Submit your manuscripts at

http://www.hindawi.com

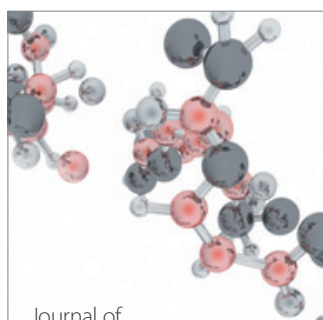

Analytical Methods

in Chemistry

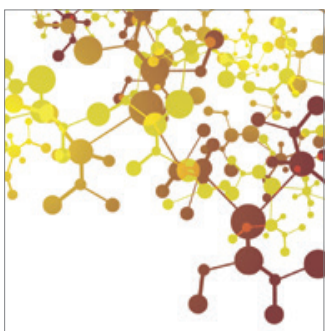

Journal of

Applied Chemistry

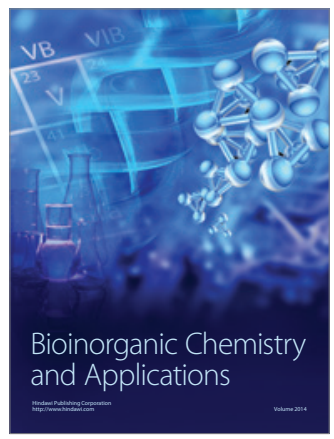

Inorganic Chemistry
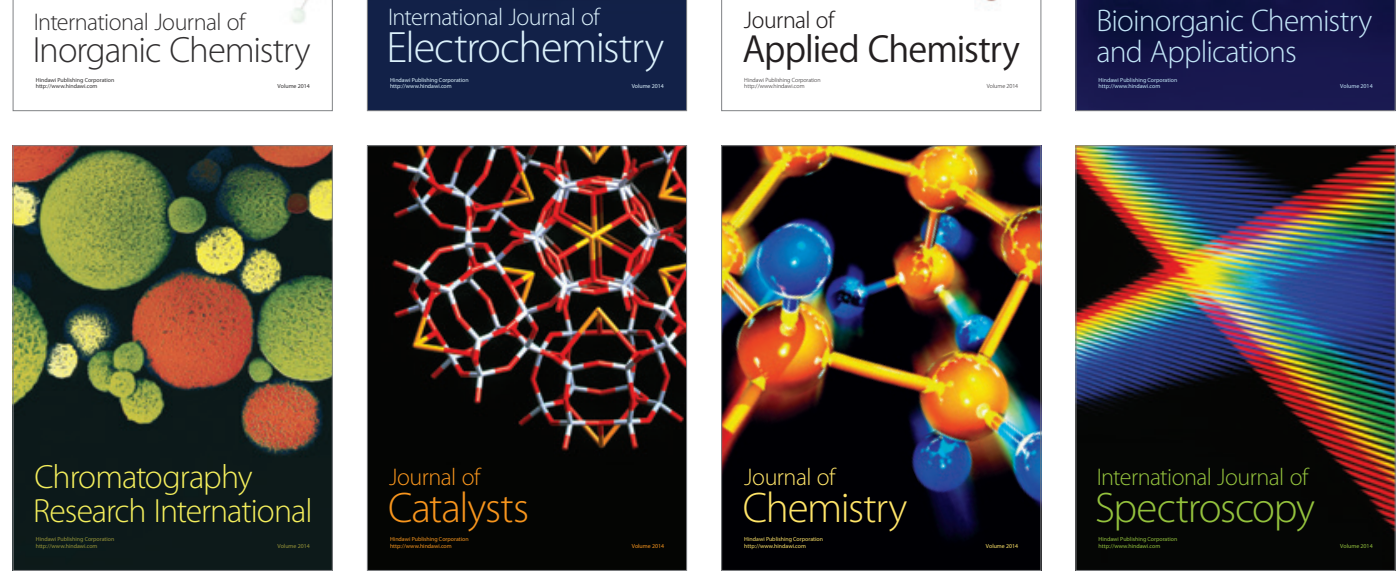